\title{
4

\section{New Analytical Approach to Nonlinear Behavior Study of Asym- metrically LCBs on Nonlinear Elastic Foundation under Steady Axial and Thermal Loading}

\begin{abstract}
In this paper, nonlinear behavior analysis of an asymmetrically laminated composite beam (LCB) on nonlinear foundation under axial and in-plane thermal loading is considered. To solve the obtained governing equation, a novel method based on Laplace transform is used. The resulted approximate analytical solution allows us the parametric study of different parameters which influence the nonlinear behavior of the system. The numerical results illustrate that proposed technique yields a very rapid convergence of the solution as well as low computational effort. The accuracy of the proposed method is verified by those available in literatures.

Keywords

Nonlinear analytical analysis; Laplace Transform; Asymmetrically Laminated Composite Beam; Thermal loading; Nonlinear elastic foundation
\end{abstract}

\section{H. Rafieipour ${ }^{*}$ A. Lotfavar and} M. H. Mansoori

Department of Mechanical and Aerospace Engineering, Shiraz University of Technology, 71555-313 Shiraz, Iran

Received 19 Apr 2012

In revised form 09 May 2012

* Author email: H.Rafieipour@stech.ac.ir Lotfavar@sutech.ac.ir

\section{INTRODUCTION}

Beam is one of the important mechanical elements and has numerous applications in different fields of engineering and industries such as civil, marine and aerospace structures or vehicles. Among these, laminated composite beams with high stiffness and strength to weight ratio are increasingly used in many engineering structures.

In most applications, they are subjected to non-linear vibrations which lead to material fatigue and structural damage due to increment of the oscillation amplitude. Therefore, it is necessary and very important to study dynamic nonlinear behavior and natural responses of these structures at large amplitudes. Furthermore, it is desirable to provide an accurate analysis towards the understanding of the non-linear vibration characteristics of these structures.

Generally, it is often difficult to find an analytical solution for a given nonlinear problem unless some simplifying assumptions are considered. Therefore, the application of different numerical techniques seems to be obligatory. It should be noted that it is hard to have a complete understanding of a nonlinear problem out of numerical results. Furthermore, numerical difficulties appear if a nonlinear problem has singularities or multiple solutions. 
However, closed form solutions are more interesting to research community even if they are approximate solutions since they have various advantages such as ease of parametric studies and considering of physics of the problem. Among approximate analytical solutions for nonlinear problems, one may refer to the homotopy perturbation method (HPM) [8], the variational iteration method (VIM) [9], the modified Lindstedt-Poincare method (MLPM)[11], the harmonic balance method (HBM) [4], the energy balance method (EBM)[20], the parameter-expansion method (PEM) [19] and He's variational method (HVM) [12].

Most studies for nonlinear vibration and buckling analysis of beams are concerned with isotropic and symmetrically LCBs, $[1,3,6,15,16]$. Due to the bending-stretching coupling in asymmetrically laminated beams, their nonlinear vibrations analyses are significantly different from that of isotropic beams and symmetrically LCBs. A few studies can be found in the literature for nonlinear analysis of asymmetrical LCBs [2, 7, 14]. For example, Patel et al.[14] used a three-nodded shear flexible beam element in order to investigate nonlinear free flexural vibrations and post-buckling of orthotropic laminated beams resting on a class of two parameter elastic foundation. Gunda et al. [7] employed the Rayleigh-Ritz method to study large amplitude vibration analysis of LCB with symmetric and asymmetric layup orientations. Baghani et al. [2] employed the variational iteration method for large amplitude free vibrations and post-buckling analysis of asymmetrically LCBs on nonlinear elastic foundation.

In this paper, geometrically nonlinear vibration and post-buckling analysis of asymmetrically LCB on nonlinear foundation under axial and in-plane thermal loading is considered. First, Galerkin method is used and the governing nonlinear partial differential equation is reduced to a single nonlinear ordinary differential equation. Afterwards, a novel method based on Laplace transform [13] that is called Laplace iteration method (LIM) is applied to obtain analytical solution for the nonlinear governing equation. Finally, an approximate analytical expression will be obtained which allows us to study effect of different parameters on nonlinear behavior of the system. In this paper for the first time, the effect of thermal loading in addition to the other effects is taking into account. The proposed technique yields very rapid convergence of the solution as well as low computational effort.

\section{SYSTEM DYNAMICS}

Consider a straight LCB of length $l$, width $b$, total thickness $h$ and mass per unit length $m$ which rests on an elastic nonlinear foundation subjected to an axial force of magnitude $\tilde{P}$ and a thermal load i.e. temperature varies linearly from $T_{b}$ at bottom side to $T_{t}$ at top side of the beam as shown in Figure 1. A Cartesian coordinate is located while its origin is at left end and its $\tilde{x}$ direction crosses through the neutral axis of the beam.

If $\tilde{w}$ and $\tilde{u}$ are the transverse and longitudinal displacements of the beam along the $\tilde{z}$ and $\tilde{x}$ directions, respectively, $\varepsilon_{0}$ shows the beam's neutral axis strain, $\kappa$ points up the flexural or bending strain of the beam which is known as the curvature and $\varepsilon_{t h}$ represents the thermal strain. Employing the Von Karman large deformation assumption, the strain-displacement relation with considering thermal effect can be shown as [10] 

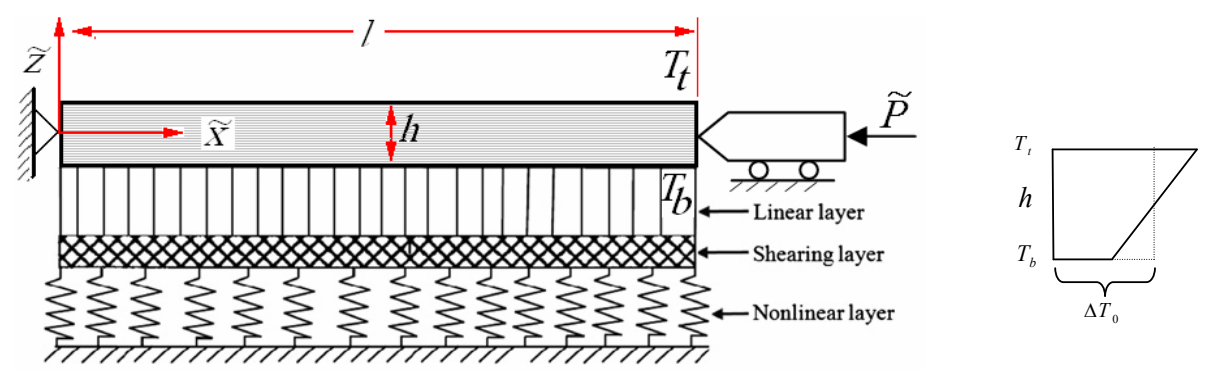

Figure 1 Schematic of the straight LCB on a nonlinear foundation and subjected to an axial force and thermal loading

$$
\varepsilon=\varepsilon_{m}+\varepsilon_{t h}
$$

where

$$
\varepsilon_{m}=\varepsilon_{0}+\tilde{z} \kappa, \quad \varepsilon_{0}=\frac{\partial \tilde{u}}{\partial \tilde{x}}+\frac{1}{2}\left(\frac{\partial \tilde{w}}{\partial \tilde{x}}\right)^{2}, \quad \kappa=-\frac{\partial^{2} \tilde{w}}{\partial \tilde{x}^{2}}, \quad \varepsilon_{t h}=\alpha_{t h} \Delta T, \quad \Delta T=\Delta T_{0}+\tilde{z} \Delta T_{1}
$$

And $\tilde{z}$ measures the distance of beam's material element from midline, $\alpha_{t h}$ is coefficient of thermal expansion. Moreover, $\Delta T_{0}$ is temperature variation at midline of the beam and $\Delta T_{1}$ stands for temperature difference between top and bottom sides and they can be presented as:

$$
\Delta T_{0}=\frac{T_{t}+T_{b}}{2} \quad, \quad \Delta T_{1}=\frac{T_{t}-T_{b}}{h}
$$

The force and moment resultants per unit length based on the classical laminate beam theory can be written as $[10,18]$ :

$$
\left\{\begin{array}{c}
N_{\tilde{x}} \\
M_{\tilde{x}}
\end{array}\right\}=\left[\begin{array}{ll}
A_{11} & B_{11} \\
B_{11} & D_{11}
\end{array}\right]\left\{\begin{array}{c}
\varepsilon_{0} \\
\kappa
\end{array}\right\}-\left[\begin{array}{ll}
A_{11 t h} & B_{11 t h} \\
B_{11 t h} & D_{11 t h}
\end{array}\right]\left\{\begin{array}{c}
\Delta T_{0} \\
\Delta T_{1}
\end{array}\right\}
$$

where its stiffness coefficients are given as follows [10, 18]:

$$
\begin{array}{ll}
A_{11}=\sum_{k=1}^{n} \bar{Q}_{11}^{(k)}\left(h_{k}-h_{k-1}\right), & A_{11 t h}=\sum_{k=1}^{n} \bar{Q}_{11}^{(k)} \alpha_{t h}^{(k)}\left(h_{k}-h_{k-1}\right) \\
B_{11}=\frac{1}{2} \sum_{k=1}^{n} \bar{Q}_{11}^{(k)}\left(h_{k}^{2}-h_{k-1}^{2}\right), & B_{11 t h}=\frac{1}{2} \sum_{k=1}^{n} \bar{Q}_{11}^{(k)} \alpha_{t h}^{(k)}\left(h_{k}^{2}-h_{k-1}^{2}\right) \\
D_{11}=\frac{1}{3} \sum_{k=1}^{n} \bar{Q}_{11}^{(k)}\left(h_{k}^{3}-h_{k-1}^{3}\right), & D_{11 t h}=\frac{1}{3} \sum_{k=1}^{n} \bar{Q}_{11}^{(k)} \alpha_{t h}^{(k)}\left(h_{k}^{3}-h_{k-1}^{3}\right)
\end{array}
$$

Each layer $k$ is referred to by the $\tilde{z}$ coordinates of its lower face $\left(h_{k-1}\right)$ and upper face $\left(h_{k}\right)$ and $\bar{Q}_{11}^{(k)}$ is the elements of the stiffness matrix in the $\tilde{x}$ direction, $n$ is the number of laminas and $\alpha_{t h}^{(k)}$ is coefficient of thermal expansion of the $k t h$ layer.

Finally, using the Extended Hamilton's principle [17, 18], the governing equation of transverse vibration of an LCB including thermal effect and axial stretching on a nonlinear elastic foundation can be obtained as 


$$
m \frac{\partial^{2} \tilde{w}}{\partial \tilde{t}^{2}}+b\left(D_{11}-\frac{B_{11}^{2}}{A_{11}}\right) \frac{\partial^{4} \tilde{w}}{\partial \tilde{x}^{4}}+\beta \frac{\partial^{2} \tilde{w}}{\partial \tilde{x}^{2}}=F_{\tilde{w}}
$$

where

$$
\begin{aligned}
& \beta=\left[\tilde{P}+b\left(A_{11 t h} \Delta T_{0}+B_{11 t h} \Delta T_{1}\right)-\frac{b A_{11}}{2 l} \int_{0}^{l}\left(\frac{\partial \tilde{w}}{\partial \tilde{x}}\right)^{2} d \tilde{x}-\frac{b B_{11}}{2 l}\left(\left.\frac{\partial \tilde{\tilde{w}}}{\partial \tilde{x}}\right|_{(l, \tilde{t})}-\left.\frac{\partial \tilde{w}}{\partial \tilde{x}}\right|_{(0, \tilde{t})}\right)\right] \\
& F_{\tilde{w}}=-\tilde{k}_{L} \tilde{w}-\tilde{k}_{N L} \tilde{w}^{3}+\tilde{k}_{S h} \frac{\partial^{2} \tilde{w}}{\partial \tilde{x}^{2}}
\end{aligned}
$$

$\tilde{k}_{L}$ and $\tilde{k}_{N L}$ are linear and nonlinear elastic foundation coefficients, $\tilde{k}_{S h}$ is the shear stiffness of the elastic foundation.

By defining non-dimensional variables

$$
x=\frac{\tilde{x}}{l}, \quad w=\frac{\tilde{w}}{r}, \quad t=\tilde{t} \sqrt{\frac{b}{m l^{4}} \gamma}, \quad r=\sqrt{\frac{I}{A}}
$$

it can be written in a simple form as

$$
\begin{aligned}
& \frac{\partial^{2} w}{\partial t^{2}}+\frac{\partial^{4} w}{\partial x^{4}}+K_{L} w+K_{N L} w^{3}-K_{S h} \frac{\partial^{2} w}{\partial x^{2}} \\
& +\left[P+F_{0 t h}+F_{1 t h}-B \int_{0}^{1}\left(\frac{\partial w}{\partial x}\right)^{2} d x-\Lambda\left(\left.\frac{\partial w}{\partial x}\right|_{(1, t)}-\left.\frac{\partial w}{\partial x}\right|_{(0, t)}\right)\right] \frac{\partial^{2} w}{\partial x^{2}}=0
\end{aligned}
$$

74

where $r$ is the radius of gyration of the beam's cross-section, and

$$
\begin{array}{ccc}
K_{L}=\frac{\tilde{k}_{L} l^{4}}{b \gamma} & K_{N L}=\frac{\tilde{k}_{N L} r^{2} l^{4}}{b \gamma} & K_{S h}=\frac{\tilde{k}_{S h} l^{2}}{b \gamma} \\
P=\frac{\tilde{P} l^{2}}{b \gamma} & F_{0 t h}=\frac{l^{2} \Delta T_{0} A_{11 t h}}{\gamma} & F_{1 t h}=\frac{l^{2} \Delta T_{1} B_{11 t h}}{\gamma} \\
B=\frac{A_{11} r^{2}}{2 \gamma} & \Lambda=\frac{B_{11} r}{\gamma} & \gamma=\left(D_{11}-\frac{B_{11}^{2}}{A_{11}}\right)
\end{array}
$$

To achieve the aims of the paper, the solution of Eq. (9) is assumed to be

$$
w(x, t)=\varphi(x) \eta(t)
$$

$$
\frac{d^{2} \eta(t)}{d t^{2}}+\left[\alpha_{1}+\left(P+F_{0 t h}+F_{1 t h}\right) \alpha_{P}+\alpha_{L}+\alpha_{S h}\right] \eta(t)+\alpha_{2} \eta^{2}(t)+\left(\alpha_{N L}+\alpha_{3}\right) \eta^{3}(t)=0
$$




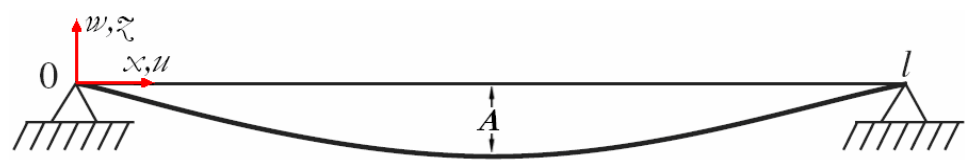

(a)

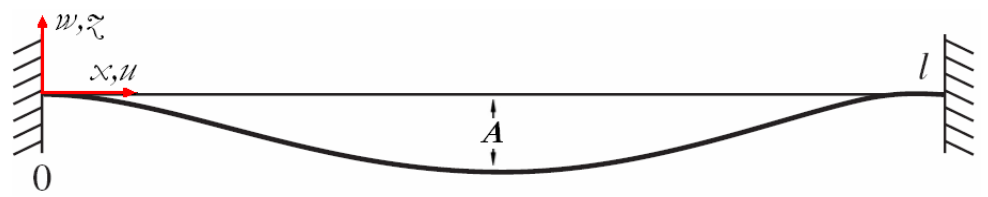

(b)

Figure 2 The first normal functions of the beam with a) Simply supported, b) Fixed-Fixed boundary conditions

where

Now, it can be assumed that the beam is subjected to an initial displacement according to its first modal shape and zero initial velocity. So, the initial conditions of Eq. (12) can be presented as

$$
\begin{aligned}
& \alpha_{1}=\frac{\int_{0}^{1} \varphi^{(i v)} \varphi d x}{\int_{0}^{1} \varphi^{2} d x}, \quad \alpha_{2}=-\Lambda\left(\varphi^{\prime}(1)-\varphi^{\prime}(0)\right) \alpha_{P}, \quad \alpha_{3}=-B \alpha_{P} \int_{0}^{1} \varphi^{\prime 2} d x \\
& \alpha_{P}=\frac{\int_{0}^{1} \varphi^{\prime \prime} \varphi d x}{\int_{0}^{1} \varphi^{2} d x}, \quad \alpha_{S h}=-K_{S h} \alpha_{P}, \quad \alpha_{L}=K_{L}, \quad \alpha_{N L}=K_{N L} \frac{\int_{0}^{1} \varphi^{4} d x}{\int_{0}^{1} \varphi^{2} d x} \\
& \eta(0)=A, \quad \frac{d \eta(0)}{d t}=0
\end{aligned}
$$

where according to the Fig. 2, $A$ denotes the non-dimensional maximum amplitude of oscillation at the beam's center.

Based on the Eq. (12) the nonlinear post-buckling load of the considered LCB can be written as

$$
P_{N B}=-\frac{\left[\alpha_{1}+\alpha_{P}+\alpha_{L}+\alpha_{S h}\right]+\alpha_{2} A+\left(\alpha_{N L}+\alpha_{3}\right) A^{2}}{\alpha_{P}}-\left(F_{0 t h}+F_{1 t h}\right)
$$

Neglecting the $A$ in Eq. (15), the linear buckling load will be derived as

$$
P_{L B}=-\frac{\alpha_{1}+\alpha_{P}+\alpha_{L}+\alpha_{S h}}{\alpha_{P}}-\left(F_{0 t h}+F_{1 t h}\right)
$$

The next step is to find the natural frequency of the system. Since the governing equation Eq. (12) is nonlinear, the free vibration of the system has a nonlinear natural frequency which is introduced by $\omega_{N L}$. Indeed, the nonlinear free vibration response of the system $\eta(t)$ and its nonlinear natural frequency $\omega_{N L}$ depend on the system parameters, the boundary condition and the initial conditions. Eq. (12) is strongly nonlinear and nobody can find an exact analytical closed form solution for $\eta(t)$ and $\omega_{N L}$. Although numerical methods can be implemented to get over this problem but, they cannot offer any suitable way for parametric 

the term $\omega^{2} u(t)$, the Eq. (17) can be written in the form

$$
\ddot{u}(t)+\omega^{2} u(t)=L\{u(t)\}=f(u(t))
$$

where $L$ is the linear operator and

$$
f(u(t))=\omega^{2} u(t)-N\{u(t)\}
$$
homogenous initial conditions gives

$$
\left(s^{2}+\omega^{2}\right) U(s)=\mathfrak{I}\{f(u(t))\}
$$
that

$$
U(s)=\mathfrak{I}\{f(u(t))\} G(s)
$$

where

$$
G(s)=\frac{1}{s^{2}+\omega^{2}}
$$

with artificial zero initial conditions and $\mathrm{N}$ is the nonlinear operator. Adding and subtracting

Taking Laplace transform of both sides of the Eq. (18) in the usual way and using the

where $s$ and $\mathfrak{I}$ are the Laplace variable and operator, correspondingly. Therefore it is obvious

where

$$
g(t)=\mathfrak{I}^{-1}\{G(s)\}=\frac{1}{\omega} \sin (\omega t)
$$

Latin American Journal of Solids and Structures 1(2012) $1-15$

Now, implementing the Laplace inverse transform of Eq. (21) and using the Convolution theorem offer

$$
u(t)=\int_{0}^{t} f(u(\tau)) g(t-\tau) d \tau
$$


Substituting Eq. (19) and (24) into (23) gives

$$
u(t)=\int_{0}^{t}\left(\omega^{2} u(\tau)-N\{u(\tau)\}\right) \frac{1}{\omega} \sin (\omega(t-\tau)) d \tau
$$

Now, the actual initial conditions must be imposed. Finally the following iteration formulation can be used [5]

$$
u_{n+1}=u_{0}+\frac{1}{\omega} \int_{0}^{t}\left(\omega^{2} u_{n}(\tau)-N\left\{u_{n}(\tau)\right\}\right) \sin (\omega(t-\tau)) d \tau
$$

Knowing the initial approximation $u_{0}$, the next approximations $u_{n}, n>0$ can be determined from previous iterations. Consequently, the exact solution may be obtained by using:

$$
u=\lim _{n \rightarrow \infty} u_{n}
$$

In this method, the problems are initially approximated with possible unknowns and it can be applied in non-linear problems without linearization or small parameters. The approximate solutions obtained by the proposed method rapidly converge to the exact solution.

\section{IMPLEMENTATION OF THE PROPOSED METHOD}

Eq. (12) can be rewritten in the standard form Eq. (18)

$$
\frac{d^{2} \eta(t)}{d t^{2}}+\omega^{2} \eta(t)=f(\eta(t))
$$

where

$$
\begin{array}{lcc}
f(\eta(t))=\omega^{2} \eta(t)-N\{\eta(t)\}, & \lambda_{1}=\alpha_{1}+\left(P+F_{0 t h}-F_{1 t h}\right) \alpha_{P}+\alpha_{L}+\alpha_{S h} \\
N\{\eta(t)\}=\lambda_{1} \eta(t)+\lambda_{2} \eta^{2}(t)+\lambda_{3} \eta^{3}(t), & \lambda_{2}=\alpha_{2}, & \lambda_{3}=\left(\alpha_{N L}+\alpha_{3}\right)
\end{array}
$$

Applying the proposed method, the following iterative formula is assembled

$$
\eta_{n+1}(t)=\eta_{0}(t)+\frac{1}{\omega} \int_{0}^{t} f\left(\eta_{n}(\tau)\right) \sin (\omega(t-\tau)) d \tau
$$

Eq. (28) will be homogeneous, if $f(\eta(t))$ is considered to be zero. So, its homogeneous solution

$$
\eta_{0}(t)=A \cos (\omega t)
$$

is considered as the zero approximation for using in iterative Eq.(30).

Expanding $f\left(\eta_{0}(\tau)\right)$, we have: 


$$
f\left(\eta_{0}(\tau)\right)=\left(-\lambda_{1} A+\omega^{2} A-\frac{3}{4} \lambda_{3} A^{3}\right) \cos (\omega t)-\frac{1}{4} \lambda_{3} A^{3} \cos (3 \omega t)-\frac{1}{2} \lambda_{2} A^{2}(1+\cos (2 \omega t))
$$

Considering the relation:

$$
\frac{1}{\omega} \int_{0}^{t}(\cos (m \omega \tau)) \sin (\omega(t-\tau)) d \tau= \begin{cases}\frac{\cos (\omega t)-\cos (m \omega t)}{\omega^{2}\left(m^{2}-1\right)} & m \neq 1 \\ \frac{t \sin (\omega t)}{2 \omega} & m=1\end{cases}
$$

To avoid secular terms in the next iterations, the coefficient of the $\cos (\omega t)$ in $f\left(\eta_{0}(\tau)\right)$ should be vanished. So the first approximation of the frequency is obtained as:

$$
\omega=\sqrt{\lambda_{1}+\frac{3}{4} \lambda_{3} A^{2}}
$$

Substituting Eq. (31) into (30) and neglecting the secular terms that are the coefficient of $\cos (\omega t)$ in forcing function $f(\eta)$ give

$$
\begin{aligned}
\eta_{1}(t)=\frac{1}{96 \omega^{2}} & \left\{\left(32 \lambda_{2} A^{2}+96 \omega^{2} A-3 \lambda_{3} A^{3}\right) \cos (\omega t)+16 \lambda_{2} A^{2} \cos (2 \omega t)\right. \\
& \left.+3 \lambda_{3} A^{3} \cos (3 \omega t)-48 \lambda_{2} A^{2}\right\}
\end{aligned}
$$

This is the first approximation of $\eta(t)$. Substituting Eq. (35) in Eq. (30) and implementing the procedure for second time yields the second approximation of $\eta(t)$ as

$$
\eta_{2}(t)=\frac{1}{1981808640} \frac{1}{A^{3}}\left(\begin{array}{l}
\mathrm{I}_{0}+\mathrm{I}_{1} \cos (\omega t)+\mathrm{I}_{2} \cos (2 \omega t)+\mathrm{I}_{3} \cos (3 \omega t) \\
+\mathrm{I}_{4} \cos (4 \omega t)+\mathrm{I}_{5} \cos (5 \omega t)+\mathrm{I}_{6} \cos (6 \omega t) \\
+\mathrm{I}_{7} \cos (7 \omega t)+\mathrm{I}_{8} \cos (8 \omega t)+\mathrm{I}_{9} \cos (9 \omega t)
\end{array}\right)
$$

where $\mathrm{I}_{i}$ are given in Appendix.

In this step, to avoid the secular terms the coefficient of $\cos (\omega t)$ in forcing function must be zero. So,

$$
\omega^{8}+\beta_{6} \omega^{6}+\beta_{4} \omega^{4}+\beta_{2} \omega^{2}+\beta_{0}=0
$$

where

$$
\begin{aligned}
& \beta_{6}=\left(-\frac{5^{2}}{2^{5}} \lambda_{3} A^{2}+\frac{1}{3} \lambda_{2} A-\lambda_{1}\right) \\
& \beta_{4}=\left(\frac{3}{2^{6}} \lambda_{3}^{2} A^{4}-\frac{3}{2^{2}} \lambda_{2} \lambda_{3} A^{3}+\left(\frac{1}{2^{5}} \lambda_{1} \lambda_{3}+\frac{5}{2 \cdot 3} \lambda_{2}^{2}\right) A^{2}-\frac{1}{3} \lambda_{1} \lambda_{2} A\right) \\
& \beta_{2}=\left(-\frac{3^{2}}{2^{12}} \lambda_{3}^{3} A^{6}+\frac{1}{2^{5}} \lambda_{2} \lambda_{3}^{2} A^{5}+\frac{5}{2 \cdot 3^{2}} \lambda_{2}^{3} A^{3}-\frac{79}{2^{5} \cdot 3} \lambda_{2}^{2} \lambda_{3} A^{2}\right) \\
& \beta_{0}=\left(\frac{3}{2^{16}} \lambda_{3}^{4} A^{8}-\frac{3}{2^{12}} \lambda_{2} \lambda_{3}^{3} A^{7}+\frac{3 \cdot 5}{2^{9}} \lambda_{2}^{2} \lambda_{3}^{2} A^{6}-\frac{5}{2^{3} \cdot 3}\right)
\end{aligned}
$$

Latin American Journal of Solids and Structures 1(2012) $1-15$ 


\section{NUMERICAL RESULTS}

To illustrate the robustness of the proposed LIM method and to compare with other methods, some cases are studied. First, an isotopic beam in two cases of simply supported and fixedfixed boundary conditions is taken. In these cases, the effects of thermal loading and elastic foundation are ignored. The amounts of the nonlinear to the linear frequency ratio $\omega_{N L} / \omega_{L}$ are derived for four non-dimensional amplitudes $A$. Table 2 shows the results of three references as well as the numerical results that are computed by the fourth Runge-Kutta method in both cases. The two last columns in each case show the results based on LIM and by one step and two step iteration. As it is mentioned, the proposed method offers the results with excellent accordance with the numerical results even by one step iteration.

Table 2 Comparison of nonlinear to linear frequency ratio, $\omega_{N L} / \omega_{L}$

\begin{tabular}{|c|c|c|c|c|c|c|c|c|c|c|c|c|}
\hline \multicolumn{7}{|c|}{ Simply Supported } & \multicolumn{6}{|c|}{ Clamped-Clamped } \\
\hline$A$ & $\begin{array}{c}\text { Ref. } \\
{[1]}\end{array}$ & $\begin{array}{l}\text { Ref. } \\
{[16]}\end{array}$ & $\begin{array}{l}\text { Ref. } \\
{[15]}\end{array}$ & $\begin{array}{c}\text { Numerical } \\
\text { results[2] }\end{array}$ & $\begin{array}{c}\text { Present } \\
1 \text { step }\end{array}$ & $\begin{array}{c}\text { Present } \\
2 \text { step }\end{array}$ & $\begin{array}{l}\text { Ref. } \\
{[1]}\end{array}$ & $\begin{array}{l}\text { Ref. } \\
{[16]}\end{array}$ & $\begin{array}{l}\text { Ref. } \\
{[15]}\end{array}$ & $\begin{array}{l}\text { Numerical } \\
\text { results [2] }\end{array}$ & $\begin{array}{c}\text { Present } \\
1 \text { step }\end{array}$ & $\begin{array}{c}\text { Present } \\
2 \text { step }\end{array}$ \\
\hline 1 & 1.0891 & 1.0897 & 1.0897 & 1.0891 & 1.0892 & 1.0892 & 1.0221 & 1.0628 & 1.0572 & 1.0553 & 1.0566 & 1.0566 \\
\hline 3 & 1.6256 & 1.6394 & 1.6393 & 1.6255 & 1.6263 & 1.6257 & 1.1831 & 1.3904 & 1.4344 & 1.4158 & 1.4179 & 1.4176 \\
\hline 4 & - & - & 1.9999 & 1.9758 & 1.9774 & 1.9761 & 1.3064 & 1.5635 & 1.6171 & 1.6658 & 1.6687 & 1.6679 \\
\hline
\end{tabular}

In the second step, it is assumed that the composite beam is made by AS4/3501 GraphiteEpoxy. Its mechanical properties [21] are $E_{11}=138 G P a, E_{22}=8.9 G P a, v_{12}=0.3, \alpha_{t h}^{(1)}=$ $-0.5 \times 10^{-6} /{ }^{\circ} \mathrm{C}$ and $\alpha_{t h}^{(2)}=28.5 \times 10^{-6} /{ }^{\circ} \mathrm{C}$. To study the effect of cross-ply lay-up configuration on the nonlinear vibration of the considered LCB, three cases of configuration are used. Figure 3 illustrates this effect on the nonlinear to the linear frequency ratio $\omega_{N L} / \omega_{L}$ and Figure 4 shows the influence on the ratio of the nonlinear post buckling load to buckling load $P_{N B} / P_{L B}$ for both cases of boundary conditions, respectively. It can be seen that the nonlinear behavior of the LCB with [0/90/90/0], [0/90/0/90] and [90/0/0/90] lay-up configuration increases from lower values to higher values, respectively. So, the LCB behavioral response can be controlled by its lay-up configuration, passively.

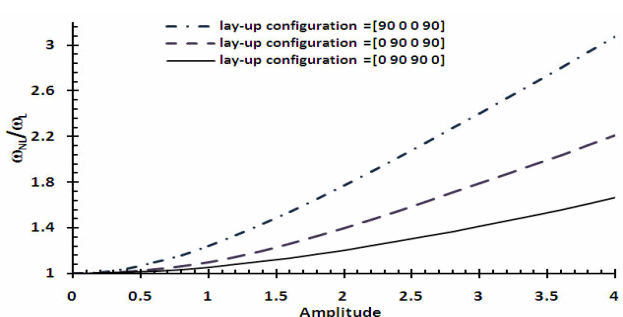

(a)

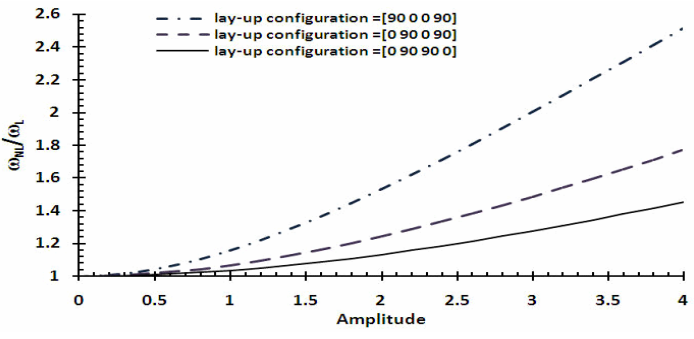

(b)

Figure 3 The effect of cross-ply lay-up configuration on the nonlinear to the linear frequency ratio Left) Simply supported, Right) Fixed-Fixed boundary conditions 


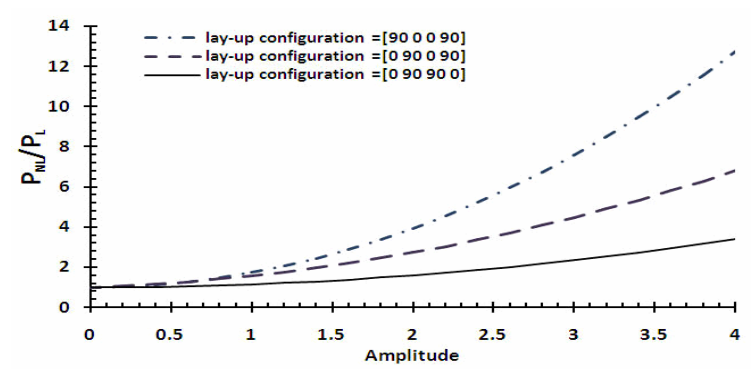

(a)

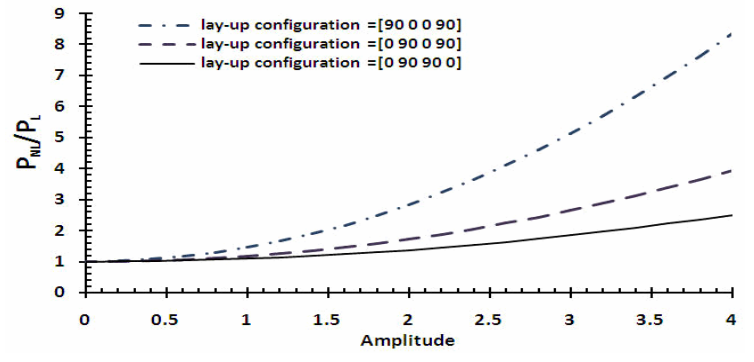

(b)

Figure 4 The effect of cross-ply lay-up configuration on the post bucking to the bucking load ratio Left) Simply supported, Right) Fixed-Fixed boundary conditions

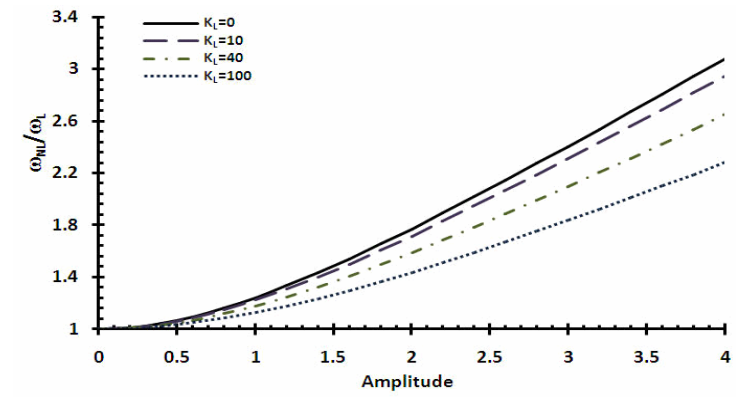

(a)

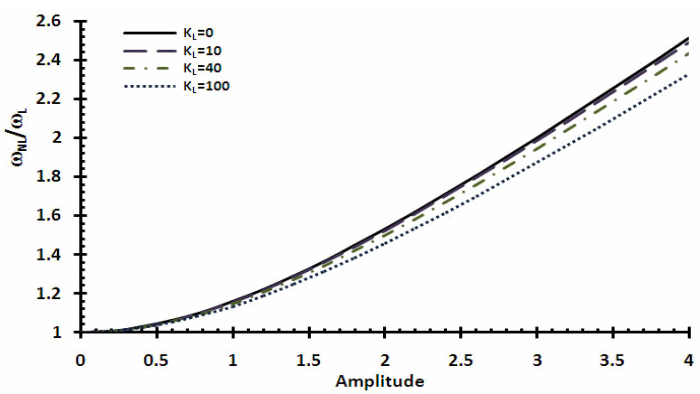

(b)

Figure 5 The effect of the linear stiffness $K_{L}$ on the nonlinear to the linear frequency ratio Left) Simply supported, Right) Fixed-Fixed boundary conditions

In the next step, the nonlinear behavior of the considered LCB due to elastic foundation is investigated. As the [90/0/0/90] lay-up has the most critical nonlinear behavior, this configuration is selected for the rest of the paper. Figure 5 to 7 demonstrate the effects of different stiffness values of $K_{L}, K_{N L}$ and $K_{S h}$ on $\omega_{N L} / \omega_{L}$ ratio for both boundary conditions, correspondingly. It can be seen that an increase in the linear and shearing layer stiffness of the foundation leads to decrement of the nonlinear to linear frequency ratio and also an increase in nonlinear stiffness augments this ratio. Also, the shearing layer stiffness has the strongest effect.

Now, the axial loading is applied. Figure 8 shows the variation of the nonlinear to the linear frequency ratio $\omega_{N L} / \omega_{L}$ due to change in the axial loading $P$. It shows that axial loading amplifies the nonlinear frequency ratio of the LCB.

Finally, the thermal loading is considered. As it is seen in Figure 9, thermal loading increases the nonlinear to the linear frequency of the considered LCB. The results show that the linear and nonlinear natural frequencies decrease by increasing the thermal loading however, the decreasing rate of nonlinear frequency is less than linear natural frequency.

In the previous steps, the effect of each factor was studied, independently. So in the last 


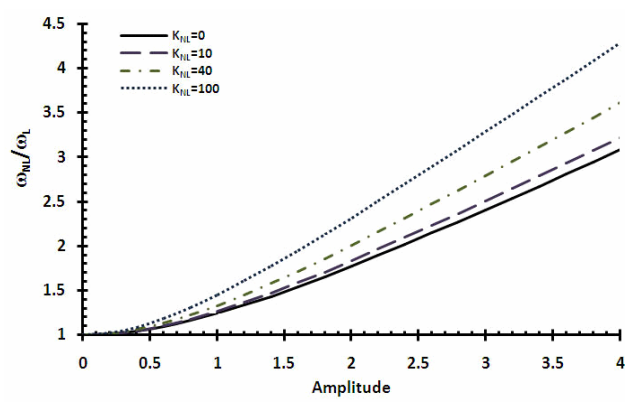

(a)

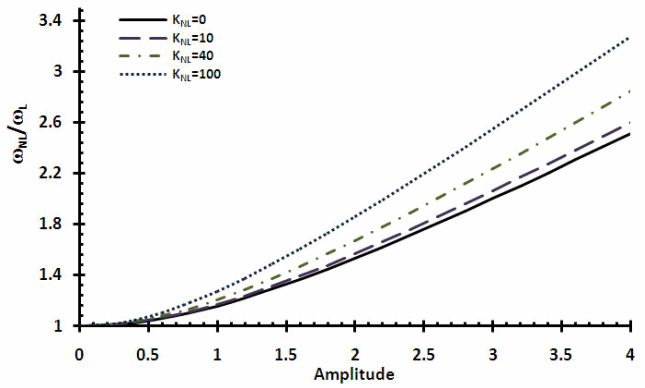

(b)

Figure 6 The effect of the nonlinear stiffness $K_{N L}$ on the nonlinear to the linear frequency ratio Left) Simply supported, Right) Fixed-Fixed boundary conditions

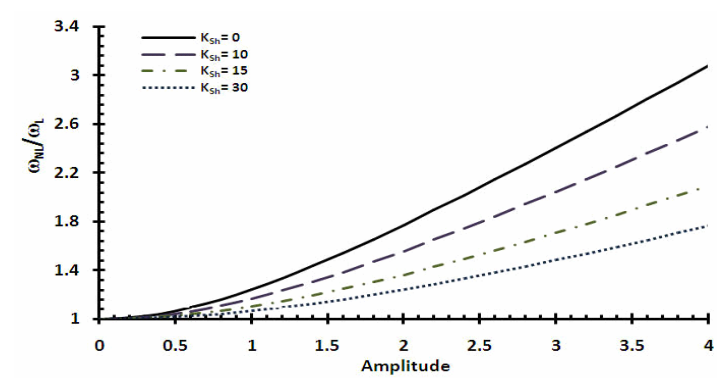

(a)

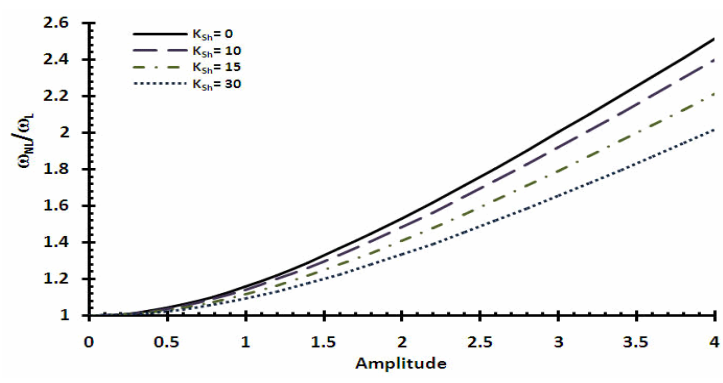

(b)

Figure 7 The effect of the shear stiffness $K_{S h}$ on the nonlinear to the linear frequency ratio Left) Simply supported, Right) Fixed-Fixed boundary conditions

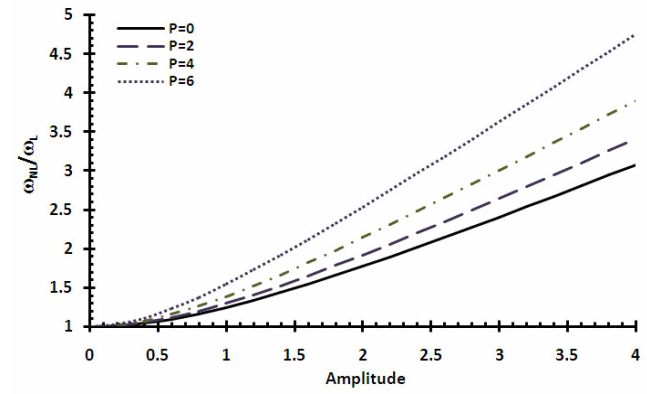

(a)

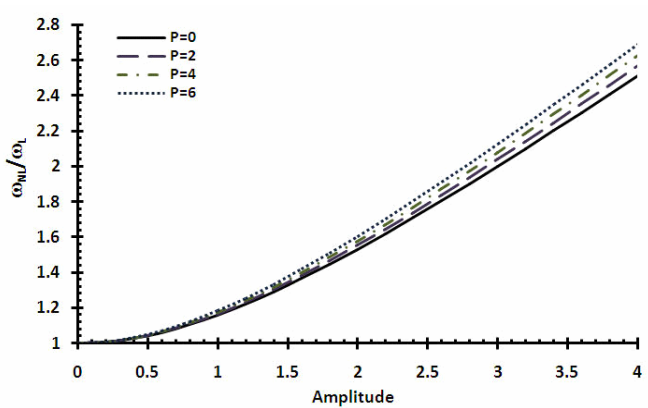

(b)

Figure 8 The effect of the axial loading on the nonlinear to the linear frequency ratio Left) Simply supported, Right) Fixed-Fixed boundary conditions 


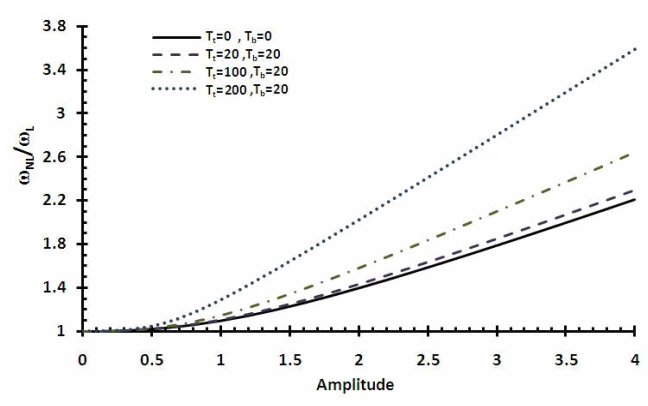

(a)

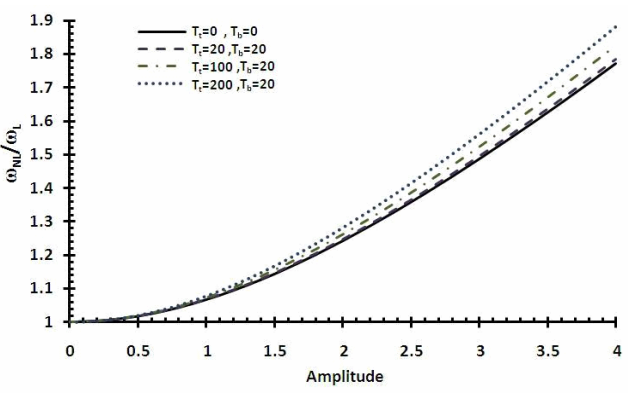

(b)

Figure 9 The effect of the thermal loading on the nonlinear to the linear frequency ratio Left) Simply supported, Right) Fixed-Fixed boundary conditions For [0/90/0/90] lay-up configuration and $\mathrm{L} / \mathrm{h}=50$

walk, effects of all factors are implemented simultaneously. Table 3 and 4 show the results for cases with simply supported and fixed-fixed boundary conditions, respectively.

Table 3 Comparison of nonlinear frequency $\left(\omega_{N L}\right)$ and nonlinear to linear frequency ratio $\left(\omega_{N L} / \omega_{L}\right)$ due to change of different factors for S-S LCB, [0/90/0/90] lay-up configuration, $A=2$ and $L / h=50$

\begin{tabular}{|c|c|c|c|c|c|c|c|c|c|c|}
\hline \multirow{3}{*}{$\left(\mathbf{T}_{t}, \mathbf{T}_{b}\right)$} & & & \multicolumn{4}{|c|}{ Nonlinear Frequency } & \multicolumn{4}{|c|}{ Nonlinear to linear Frequency } \\
\hline & \multirow[t]{2}{*}{$\mathbf{P}$} & \multirow[t]{2}{*}{$\mathbf{K}_{L}$} & \multicolumn{4}{|l|}{$\mathbf{K}_{N L}$} & \multicolumn{4}{|l|}{$\mathbf{K}_{N L}$} \\
\hline & & & $\mathbf{0}$ & \multicolumn{3}{|c|}{50} & $\mathbf{0}$ & \multicolumn{3}{|c|}{50} \\
\hline & & & $\mathrm{K}_{s h}$ & \multicolumn{3}{|c|}{$\mathrm{K}_{s h}$} & $\mathrm{~K}_{s h}$ & \multicolumn{3}{|c|}{$\mathrm{K}_{s h}$} \\
\hline & & & 0 & 25 & 0 & 25 & 0 & 25 & 0 & 25 \\
\hline \multirow[t]{4}{*}{$(0,0)$} & 0 & 0 & 13.796 & 20.771 & 17.622 & 23.439 & 1.3978 & 1.1197 & 1.7855 & 1.2635 \\
\hline & & 50 & 15.423 & 21.935 & 18.919 & 24.468 & 1.2703 & 1.1049 & 1.5583 & 1.2325 \\
\hline & 3 & 0 & 12.771 & 20.051 & 16.821 & 22.810 & 1.5510 & 1.1306 & 2.0428 & 1.2862 \\
\hline & & 50 & 14.475 & 21.253 & 18.159 & 23.864 & 1.3336 & 1.1131 & 1.6730 & 1.2499 \\
\hline \multirow[t]{4}{*}{$(50,50)$} & 0 & 0 & 13.047 & 20.244 & 17.036 & 22.978 & 1.5001 & 1.1275 & 1.9587 & 1.2798 \\
\hline & & 50 & 14.731 & 21.436 & 18.363 & 24.025 & 1.3142 & 1.1108 & 1.6382 & 1.2450 \\
\hline & 3 & 0 & 11.994 & 19.506 & 16.217 & 22.337 & 1.7676 & 1.1400 & 2.3900 & 1.3054 \\
\hline & & 50 & 13.749 & 20.738 & 17.585 & 23.411 & 1.4030 & 1.1201 & 1.7944 & 1.2645 \\
\hline \multirow[t]{4}{*}{$(150,50)$} & 0 & 0 & 11.935 & 19.464 & 16.171 & 22.301 & 1.7915 & 1.1408 & 2.4273 & 1.3071 \\
\hline & & 50 & 13.693 & 20.698 & 17.541 & 23.376 & 1.4095 & 1.1206 & 1.8055 & 1.2656 \\
\hline & 3 & 0 & 10.864 & 18.696 & 15.328 & 21.642 & 2.8264 & 1.1561 & 3.9877 & 1.3383 \\
\hline & & 50 & 12.664 & 19.976 & 16.738 & 22.745 & 1.5735 & 1.1318 & 2.0797 & 1.2887 \\
\hline
\end{tabular}

Latin American Journal of Solids and Structures 1(2012) $1-15$ 
Table 4 Comparison of nonlinear frequency $\left(\omega_{N L}\right)$ and nonlinear to linear frequency ratio $\left(\omega_{N L} / \omega_{L}\right)$ due to change of different factors for F-F LCB, [0/90/0/90] lay-up configuration, $A=2$ and $L / h=50$

\begin{tabular}{|c|c|c|c|c|c|c|c|c|c|c|}
\hline \multirow{3}{*}{$\left(\mathbf{T}_{t}, \mathbf{T}_{b}\right)$} & \multirow{3}{*}{$\mathbf{P}$} & \multirow{3}{*}{$\mathbf{K}_{L}$} & \multicolumn{4}{|c|}{ Nonlinear Frequency } & \multicolumn{4}{|c|}{ Nonlinear to linear Frequency } \\
\hline & & & $\begin{array}{c}\mathbf{K}_{N L} \\
\mathbf{0} \\
\mathrm{K}_{s h}\end{array}$ & \multicolumn{3}{|c|}{$\mathbf{5 0}$} & $\begin{array}{c}\mathbf{K}_{N L} \\
\mathbf{0} \\
\mathrm{K}_{s h}\end{array}$ & \multicolumn{3}{|c|}{$\begin{array}{c}50 \\
\mathrm{~K}_{s h}\end{array}$} \\
\hline & & & 0 & 25 & 0 & 25 & 0 & 25 & 0 & 25 \\
\hline \multirow[t]{4}{*}{$(0,0)$} & 0 & 0 & 27.778 & 32.868 & 32.425 & 36.899 & 1.2416 & 1.1562 & 1.4493 & 1.2980 \\
\hline & & 50 & 28.668 & 33.622 & 33.195 & 37.575 & 1.2218 & 1.1477 & 1.4147 & 1.2827 \\
\hline & 3 & 0 & 27.102 & 32.300 & 31.844 & 36.392 & 1.2586 & 1.1631 & 1.4788 & 1.3104 \\
\hline & & 50 & 28.014 & 33.067 & 32.628 & 37.077 & 1.2361 & 1.1539 & 1.4396 & 1.2938 \\
\hline \multirow[t]{4}{*}{$(50,50)$} & 0 & 0 & 27.283 & 32.451 & 31.999 & 36.527 & 1.2539 & 1.1612 & 1.4706 & 1.3071 \\
\hline & & 50 & 28.189 & 33.215 & 32.779 & 37.210 & 1.2321 & 1.1522 & 1.4327 & 1.2908 \\
\hline & 3 & 0 & 26.594 & 31.876 & 31.410 & 36.015 & 1.2729 & 1.1686 & 1.5034 & 1.3203 \\
\hline & & 50 & 27.523 & 32.653 & 32.205 & 36.707 & 1.2478 & 1.1587 & 1.4601 & 1.3026 \\
\hline \multirow[t]{4}{*}{$(150,50)$} & 0 & 0 & 26.555 & 31.844 & 31.376 & 35.986 & 1.2740 & 1.1690 & 1.5053 & 1.3211 \\
\hline & & 50 & 27.485 & 32.621 & 32.173 & 36.679 & 1.2487 & 1.1591 & 1.4617 & 1.3033 \\
\hline & 3 & 0 & 25.846 & 31.257 & 30.775 & 35.466 & 1.2963 & 1.1771 & 1.5435 & 1.3356 \\
\hline & & 50 & 26.802 & 32.049 & 31.587 & 36.169 & 1.2669 & 1.1663 & 1.4931 & 1.3162 \\
\hline
\end{tabular}

\section{CONCLUSION}

In this paper, the effects of different parameters such as vibration amplitude, nonlinear elastic foundation, axial and thermal loading on the nonlinear behavior of the LCBs such as natural frequency and buckling load were investigated. For this purpose and to solve nonlinear governing equation, a new approach based on the Laplace transform method which is called LIM was implemented. This technique provides the ability for parametric study of the considered problem. Results revealed that the presented method offers accurate solution with low computational effort.

Moreover, the presented expression is valid for a wide range of vibration amplitudes while predictions of the other analytical techniques such as perturbation methods are valid for small amplitudes. Comparison between the results of the present study and other methods available in the literature shows the accuracy of the method. Results reveal that decreasing linear and shear parameters and increasing nonlinear parameters of foundation lead to increasing frequency and buckling load ratios. Furthermore, increasing axial force decreases absolute values of both linear and nonlinear frequencies as well as natural frequency ratio.

Appendix: $I_{i}$ coefficients in the second approximation of deflection.

$$
\begin{array}{r}
\mathrm{I}_{0}=990904320 \mathrm{~A}\left(\left(\Pi_{1}+\frac{21}{16} \Pi_{3}-2 \Pi_{4}\right) \mathrm{A}^{2}+\left(-\frac{1}{32} \Pi_{3}^{2}+\left(-\frac{49}{512} \Pi_{5}+\frac{41}{48} \Pi_{4}\right) \Pi_{3}-\frac{2}{3} \Pi_{4}^{2}\right)\right. \\
\left.\mathrm{A}+\left(\frac{13}{4096} \Pi_{5}^{2}+\frac{31}{72} \Pi_{4}^{2}\right) \Pi_{3}-\frac{23}{36} \Pi_{4}^{3}\right)
\end{array}
$$

$\mathrm{I}_{1}=1981808640 \mathrm{~A}^{4}+\left(-54190080 \Pi_{5}-1052835840 \Pi_{3}-1101004800 \Pi_{1}-7741440 \Pi_{2}+1761607680 \Pi_{4}\right) \mathrm{A}^{3}$

$+\left(26512128 \Pi_{3}^{2}+\left(-595574784 \Pi_{4}+101007360 \Pi_{5}\right) \Pi_{3}-7741440 \Pi_{5}^{2}+399114240 \Pi_{4}^{2}\right) \mathrm{A}^{2}+$

$\left(\left(-457900032 \Pi_{4}^{2}-3740640 \Pi_{5}^{2}\right) \Pi_{3}+690880512 \Pi_{4}^{3}+574560 \Pi_{5}^{3}\right) \mathrm{A}-14364 \Pi_{5}^{4}$ 


$$
\begin{array}{r}
\mathrm{I}_{2}=110100480 \mathrm{~A}\left(\left(\Pi_{1}-3 \Pi_{3}+2 \Pi_{4}\right) \mathrm{A}^{2}+\left(-\frac{1}{64} \Pi_{3}^{2}+\left(-2 \Pi_{4}-\frac{51}{1024} \Pi_{5}\right) \Pi_{3}+2 \Pi_{4}^{2}\right)\right. \\
\left.\mathrm{A}+\left(\frac{7}{16} \Pi_{4}^{2}+\frac{3}{512} \Pi_{5}^{2}\right) \Pi_{3}-\frac{2}{3} \Pi_{4}^{3}\right)
\end{array}
$$

$$
\begin{aligned}
& \mathrm{I}_{3}=\left(7741440 \Pi_{2}+54190080 \Pi_{5}+61931520 \Pi_{3}\right) \mathrm{A}^{3}+\left(\begin{array}{l}
8547840 \Pi_{3}^{2}+\left(3870720 \Pi_{5}-45158400 \Pi_{4}\right) \Pi_{3} \\
+5806080 \Pi_{5}^{2}+41287680 \Pi_{4}^{2}
\end{array}\right) \mathrm{A}^{2} \\
& +\left(\left(-181440 \Pi_{5}^{2}-16629760 \Pi_{4}^{2}\right) \Pi_{3}-544320 \Pi_{5}^{3}+13762560 \Pi_{4}^{3}\right) \mathrm{A}+15120 \Pi_{5}^{4}
\end{aligned}
$$

$$
\begin{gathered}
\mathrm{I}_{4}=20643840 \mathrm{~A}\left(\mathrm{~A}^{2} \Pi_{3}-\frac{1}{10} \Pi_{3}\left(\frac{49}{16} \Pi_{5}-6 \Pi_{4}+\Pi_{3}\right) \mathrm{A}+\left(-\frac{2}{45} \Pi_{4}^{2}+\frac{3}{320} \Pi_{5}^{2}\right) \Pi_{3}+\frac{4}{45} \Pi_{4}^{3}\right) \\
\mathrm{I}_{5}=-483840 \mathrm{~A}\left(\left(\Pi_{3}^{2}+\left(-\frac{8}{3} \Pi_{5}-\frac{40}{9} \Pi_{4}\right) \Pi_{3}-4 \Pi_{5}^{2}\right) \mathrm{A}+\left(-\frac{32}{27} \Pi_{4}^{2}+\frac{\Pi_{5}^{2}}{24}\right) \Pi_{3}+\frac{\Pi_{5}^{3}}{8}\right) \\
\mathrm{I}_{6}=147456 \mathrm{~A} \Pi_{3}\left(\left(\Pi_{3}+\frac{51}{16} \Pi_{5}\right) \mathrm{A}-\frac{3}{8} \Pi_{5}^{2}+\frac{4}{9} \Pi_{4}^{2}\right)
\end{gathered}
$$

$$
\mathrm{I}_{7}=26880 \Pi_{3}^{2} \mathrm{~A}^{2}+10080 \Pi_{5}^{2}\left(3 \Pi_{5}+\Pi_{3}\right) \mathrm{A}-945 \Pi_{5}^{4} \quad \mathrm{I}_{8}=3840 \Pi_{5}^{2} \mathrm{~A} \Pi_{3} \quad \mathrm{I}_{9}=189 \Pi_{5}^{4}
$$

Where

$$
\Pi_{1}=\frac{\lambda_{1} A^{2} \lambda_{2}}{\omega^{4}}, \quad \Pi_{2}=\frac{\lambda_{1} A^{3} \lambda_{3}}{\omega^{4}}, \quad \Pi_{3}=\frac{\lambda_{2} A^{4} \lambda_{3}}{\omega^{4}}, \quad \Pi_{4}=\frac{\lambda_{2} A^{2}}{\omega^{2}}, \quad \Pi_{5}=\frac{\lambda_{3} A^{3}}{\omega^{2}}
$$

\section{References}

[1] L. Azrar, R. Benamar, and R.G. White. A semi-analytical approach to the nonlinear dynamic response problem of s-s and c-c beams at large vibration amplitudes, part i: general theory and application to the single mode approach to free and forced vibration analysis. J Sound Vib, 224:183-207, 1999.

[2] M. Baghani, R.A. Jafari Talookolaei, and H. Salarieh. Large amplitudes free vibrations and post-buckling analysis of unsymmetrically laminated composite beams on nonlinear elastic foundation. Appl Math Model, 35:130-138, 2011.

[3] A. Barari. Non-linear vibration of euler-bernoulli beams. Latin American Journal of Solids and Structures, 8(2):139$148,2011$.

[4] A. Beléndez, A. Hernandez, T. Beléndez, M.L. Álvarez, S. Gallego, M. Ortu no, and C. Neipp. Application of the harmonic balance method to a nonlinear oscillator typified by a mass attached to a stretched wire. J Sound Vib, 302:1018-1029, 2007.

[5] E.Hesameddini and H. Latifizadeh. Reconstruction of variational iteration algorithms using the laplace transform. Int J Nonlinear Sci Numer Simul, 10(10):1365-1370, 2009.

[6] S.A. Emam. A static and dynamic analysis of the post buckling of geometrically imperfect composite beams. Compos Struct, 90:247-253, 2009.

[7] J.B. Gunda, R.K. Gupta, G.R. Janardhan, and G.V. Rao. Large amplitude vibration analysis of composite beams: simple closed-form solutions. Compos Struct, 93:870-879, 2010.

[8] JH. He. Homotopy perturbation method for solving boundary value problems. Phys Lett A, 350:87-88, 2006. 
[9] JH. He. Variational iteration method: some recent results and new interpretations. J Comput Appl Math, 207:3-17, 2007.

[10] L.P. Kollar and G.S. Springer. Mechanics of composite structures. Cambridge University Press, 2003.

[11] HM. Liu. Approximate period of nonlinear oscillators with discontinuities by modified lindstedt-poincare method. Chaos Solitons Fractals, 23:577-579, 2005.

[12] J.F. Liu. He's variational approach for nonlinear oscillators with high nonlinearity. Comput Math Appl, 58:2423-2426, 2009.

[13] A. Lotfavar, H. Rafieipour, and H. Latifizadeh. Application of the general variational iteration method to a nonlinear system. In The 2011 International Conference of Applied and Engineering Mathematics, ICAEM-127, volume 6, pages 182-185, London, July 2011.

[14] B.P. Patel, M. Ganapathi, and M. Touratier. Nonlinear free flexural vibrations/post-buckling analysis of laminated orthotropic beams/columns on a two parameter elastic foundation. Compos Struct, 46:189-196, 1999.

[15] T. Pirbodaghi, M.T. Ahmadian, and M. Fesanghary. On the homotopy analysis method for nonlinear vibration of beams. Mech Res Commun, 36(2):143-148, 2009.

[16] M.I. Qaisi. Application of the harmonic balance principle to the nonlinear free vibration of beams. Appl Acoust, 40:141-151, 1993.

[17] S. S. Rao. Vibration of Continuous Systems. John Wiely \& Sons, New Jersy, 2007.

[18] J. Reddy. Mechanics of Laminated Composite Plates Theory and Analysis. CRC, Boca Raton, 1997.

[19] D.H. Shou and JH. He. Application of parameter-expanding method to strongly nonlinear oscillators. Int J Nonlin Sci Numer Simul, 8:121-124, 2007.

[20] D. Younesian, H. Askari, Z. Saadatnia, and M. KalamiYazdi. Frequency analysis of strongly nonlinear generalized duffing oscillators using he's frequency amplitude formulation and he's energy balance method. Comput Math Appl, $59: 3222-3228,2010$.

[21] F.G. Yuan. Thermal stresses in thick laminated composite shells. Composite Structures, 26:63-75, 1993. 
